\title{
SUPPLEMENTARY MATERIAL ACCOMPANYING:
}

Comparison of Approximate Methods for Calculating the Friction Coefficient and Intrinsic Viscosity of Nanoparticles and Macromolecules

\author{
Marc L. Mansfield ${ }^{1 *}$, Jack F. Douglas ${ }^{2 *}$, Saba Irfan ${ }^{l}$, Eun-Hee Kang ${ }^{l}$ \\ ${ }^{1}$ Department of Chemistry and Chemical Biology, Stevens Institute of Technology, \\ Hoboken, New Jersey 07030 \\ ${ }^{2}$ Polymers Division, National Institute of Standards and Technology, Gaithersburg, \\ Maryland 20899
}

We have employed a Monte Carlo integration technique to evaluate $R_{K}$, the "Kirkwood radius," and $A$, the surface area, of a number of different surfaces. This document describes the approach.

All of the surfaces examined in this paper are constructed from a set of "building blocks" which are themselves simple surfaces. We classify these building blocks as either open or closed surfaces, where by 'closed surface' we simply mean a surface that encloses a region of three-dimensional space. The open surfaces include triangles, circular disks, and open cylinders. (An open cylinder is a tin can with both ends cut off, while a closed cylinder has the ends intact.) The closed surfaces include spheres, cubes, tori and ellipsoids.

Compound surfaces are formed from these simple components, with the possibility that components overlap. When they do, we assume that the complete surface 
consists of all those points on any one of the component surfaces that do not lie inside some other closed component surface. For example, we have represented protein molecules as unions of spheres of radius $5 \AA$, one sphere centered at each $\alpha$-carbon. ${ }^{14}$ Since $\alpha$-carbons can lie within $5 \AA$ of one another, the resulting spheres overlap. In such a case, the surface is defined as the set of all points lying on the surface of any one of the spheres, but not in the interior of any other sphere. As other examples, we have modeled closed cylinders as the union of one open cylinder and two circular disks, and a number of complex surfaces with a triangular mesh. ${ }^{13}$

To evaluate the integral of Eq. (10), we need to generate points at random over the surface. As mentioned above, the complete surface is assumed to be composed of $N$ component surfaces, each one of which can be a triangle, a circular disk, an open cylinder, or the surface of a sphere, cube, torus, or ellipsoid. Let $S_{j}$ represent the surface area of the j-th component surface and we then introduce the definition, $S_{0}=\sum_{j} S_{j}$. To obtain a random point on the compound surface, we first select at random one of the $N$ component surfaces, with the selection probability of surface $j$ defined as, $S_{j} / S_{0}$. Then, a point is selected uniformly and at random on this component surface. If the resulting point lies inside any other closed component surface, then it is rejected, and the above procedure is performed again. This repeats until contributions from a large number of points have been accumulated. The surface area of the compound surface, needed for evaluation of Eqs. (15) and (16), is estimated as $S_{0}$ times the fraction of points not rejected. The Kirkwood radius is calculated as the harmonic mean distance between successive pairs of points. We must still address two simpler problems: first, generating points distributed randomly over any of the $S_{j}$, and second, computing the area of $S_{j}$, 
where $S_{j}$ represents one of the component surfaces or its area. These two problems are discussed in the following paragraphs.

First, we consider the problem of generating points distributed randomly over each of the component surfaces listed above. Given a standard random number generator, it is, of course, a trivial matter to generate points distributed over the interior of either a rectangle or a cube, and one or the other of these is our starting point in each case. The precise algorithm followed for each of the simple surfaces is described in the following paragraphs.

Triangles. One of the three sides of the triangle is arbitrarily chosen as the base. Then the triangle is enclosed in a rectangle, with one side parallel to and containing the base, the other side parallel to the altitude. A tentative point is selected at random somewhere in the interior of this rectangle, and retained if it also lies inside the triangle. If not, it is discarded and another is selected.

Circular disks. Points are generated at random inside a square in which the circle is inscribed, and retained if they also lie inside the circle.

Open cylinders. The open cylinder is a rolled-up rectangle. Points are generated on the rectangle and then mapped to the cylinder.

$\underline{\text { Spheres. }}$ Points are generated randomly over the interior of a unit cube, retained if they also lie inside the unit sphere, and then projected along the ray from the center onto the surface of the sphere.

Cubes. Points are generated randomly over the interior of a square. Then another random number is generated and used to select one of the six faces, to which the point is then mapped. 
Tori. We regard the torus as a deformed cylinder, and begin by generating a point on the cylinder. When the cylinder is mapped to the torus, however, some parts of the surface are stretched more than others, so that a uniform distribution on the cylinder is not mapped uniformly onto the torus. We compensate for this by discarding points with probability: $1-\frac{\sigma}{\sigma_{\max }}$, where $\sigma$ is the local stretching factor, and $\sigma_{\max }$ is the maximum value attained by $\sigma$ over the cylinder. More specifically, we define the torus as the body generated by revolving a circle of radius $r_{2}$ around an external axis a distance $r_{1}$ from the center of the circle. Then we generate two random angles, $\theta \in[0,2 \pi]$ and $\varphi \in[0,2 \pi]$. Assuming a Cartesian coordinate system for which the z-axis coincides with the symmetry axis of the torus, we begin by placing a point at $\left(r_{2} \cos \theta+r_{1}, 0, r_{2} \sin \theta\right)$ and then we rotate this point about the z-axis through the angle $\varphi$. The stretching factor for this point is $\sigma=r_{1}+r_{2} \cos \theta$, and the maximum occurs when $\theta=0: \sigma_{\max }=r_{1}+r_{2}$.

Ellipsoids. We assume a local rectangular coordinate system for the ellipsoid, such that the ellipsoid is determined by the equation

$$
\left(\frac{x}{a}\right)^{2}+\left(\frac{y}{b}\right)^{2}+\left(\frac{z}{c}\right)^{2}=1
$$

with $a \geq b \geq c$. A point $(x, y, z)$ is generated randomly on the surface of the unit sphere, as explained above. Then it is mapped to the point $(a x, b y, c z)$. But once again, because of non-uniform stretching, it may be necessary to discard this point. The local stretching factor, $\sigma$, is computed as follows. Note, first of all, that the transformation $(x, y, z) \rightarrow(a x, b y, c z)$ is equivalent to operation on $(x, y, z)$ with a second-rank symmetric tensor, diagonal in this reference frame, which we designate $\mathbf{A}$. 
Let $\hat{\mathbf{n}}=(x, y, z)$ represent the point on the surface of the sphere. We then map it to spherical-polar coordinates: $\hat{\mathbf{n}}=(\sin \theta \cos \varphi, \sin \theta \sin \varphi, \cos \theta)$. We imagine that the surface is stretched locally along two separate unit vectors:

$\hat{\mathbf{m}}=(\cos \theta \cos \varphi, \cos \theta \sin \varphi,-\sin \theta)$, which is tangent to the sphere in the direction of increasing $\theta$; and $\hat{\mathbf{k}}=(-\sin \varphi, \cos \varphi, 0)$, which is tangent in the direction of increasing $\varphi$. Then the local stretching factor is the product of the factors in each of the two directions: $\sigma=|\mathbf{A} \cdot \hat{\mathbf{m}}||\mathbf{A} \cdot \hat{\mathbf{k}}|$. The maximum stretching factor for ellipsoids is obtained as follows. The two unit vectors $\hat{\mathbf{m}}$ and $\hat{\mathbf{k}}$ are always orthogonal, and the maximum $\sigma$ occurs when they both lie in the plane spanned by the two dominant eigenvectors of $\mathbf{A}$, since even a slight deformation out of this plane mixes in contributions from the smallest eigenvalue, $c$. It is not difficult to show that the maximum occurs when $\hat{\mathbf{m}}$ and $\hat{\mathbf{k}}$ make angles of $\pi / 4$ with the two eigenvectors in this plane, and that

$$
\sigma_{\max }=\left[a^{2} b^{2}+\frac{1}{4}\left(a^{2}-b^{2}\right)^{2}\right]^{\frac{1}{2}}
$$

Then, as before, the point is discarded with probability, $1-\frac{\sigma}{\sigma_{\max }}$.

As mentioned above, we must also compute the total surface area of each of the component surfaces. For all but the ellipsoid, the surface area formulas are well-known. For ellipsoids, we determine the total surface area by integrating $\sigma$ over the original surface,

$$
S=\int_{0}^{\pi} \sin \theta d \theta \quad \int_{0}^{2 \pi} d \varphi \quad|\mathbf{A} \cdot \hat{\mathbf{m}}| \mathbf{A} \cdot \hat{\mathbf{k}} \mid
$$


In the general case, $a \neq b \neq c$, Eq. (S3) involves elliptic integrals, so we have evaluated it numerically. 